Research Paper:

\title{
Balance Performance With and Without Visual Information: A Comparison of Elite Players With Chronic Ankle Instability
}

\author{
${\text { Zahra Raeisi }{ }^{1^{*}} \text { (D) , Ali Yalfani }}^{2}$ (iD)
}

1. Department of Sport Injuries and Corrective Exercises, Faculty of Sport Science, Arak University, Arak, Iran.

2. Department of Sports Injuries and Corrective Exercises, Faculty of Sport Science, University of Bu-Ali Sina, Hamedan, Iran.

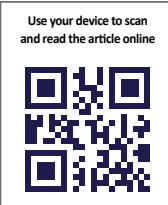

Citation Raeisi Z, Yalfani A. Balance Performance With and Without Visual Information: A Comparison of Elite Players With Chronic Ankle Instability. Journal of Exercise Science and Medicine (JESM). 2019; 11(2):125-132. http://dx.doi.org/10.32598/ JESM.11.2.8

ittp://dx.doi.org/10.32598/JESM.11.2.8

\section{(c) (i) (5)}

Article info:

Received: 10 Jan 2019

Accepted: 23 May 2019

Available Online: $01 \mathrm{Jul} 2019$

Keywords:

Postural control, Chronic ankle instability, Soccer, Volleyball, Basketball

\begin{abstract}
A B S T R ACT
Introduction: This research was conducted to determine whether professional athletes with Chronic Ankle Instability (CAI) exhibit differences in maintaining balance under conditions of with and without visual information

Materials and Methods: Forty-five elite players with CAI were classified into soccer, volleyball, and basketball groups ( $\mathrm{n}=15$ each). All participants performed a static single-leg balance test in conditions wherein visual information was provided and withheld. Also, their Center of Pressure (CoP) oscillation parameters was measured. The data were examined through an Analysis of Variance (ANOVA) and the Tukey test.

Results: A significant difference was found between the soccer and volleyball players $(P=0.034)$ in anteroposterior CoP displacement. Furthermore, there was a significant difference between soccer compared with volleyball $(P=0.001)$ and basketball $(P=0.02)$ groups regarding mediolateral sway and sway area $(P=0.003$ and $P=0.03$, respectively) when they performed the test with their eyes open. A significant difference occurred between the soccer and volleyball players concerning anteroposterior and mediolateral sway $(\mathrm{P}=0.002$ and $\mathrm{P}=0.04$, respectively $)$ and sway area $(\mathrm{P}=0.004)$ when they performed the test with their eyes closed.

Conclusion: Given the necessity of maintaining a good balance in sports, volleyball coaches should design practices that reduce players' reliance on visual information. These practices should strengthen their proprioceptive abilities through an increased challenge to their proprioceptive senses.
\end{abstract}

\section{* Corresponding Author:}

Zahra Raeisi, PhD.

Address: Department of Sport Injuries and Corrective Exercises, Faculty of Sport Science, Arak University, Arak, Iran.

Tel: +98 (913) 3848636

E-mail: z_raisi13@yahoo.com 


\section{Introduction}

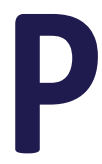

ostural control or balance is essential in the independent performance of daily activities, such as walking, climbing up and down the stairs, and standing on a bus [1]. In addition to ensuring effective engagement in everyday routines, balance plays a crucial role in completing complex tasks, including sport-related activities [2]. Postural control skills also determine successful performance- a fact that has recently been adopted in the domain of sports [3]. For balance control, the Central Nervous System (CNS) integrates visual, vestibular, and proprioceptive information to generate motor commands that coordinate muscle activation patterns [4]. Balance, which is often used to measure lower limb function, is defined as a process of maintaining the center of gravity at the level of the body's base of support [5]. Balance and coordination are core fitness competencies in many sports $[3,6,7]$. The emphasis on balance in sports activities is due to the association between postural control and the risk of sports-related injuries [7]. In this regard, the ankle is the most commonly injured part of the body among elite players of team ball sports, such as volleyball, basketball, and soccer [8].

This injury is related considerably to reducing sensory and motor control (e.g. proprioception), muscle strength, and balance function [9]. Ankle sprains alter proprioception and balance by damaging the cutaneous, muscular, tendon, and joint receptors as well as gamma motor neurons. Sensorimotor disorders are likewise associated with lower extremity injuries and thereby causing dysfunction and increasing the risk of re-injury [9]. The recurrence rate of ankle sprains is as high as $70 \%$ and often leads to Chronic Ankle Instability (CAI), which is generally associated with frequent injuries as well as sports and participation restrictions [10].

According to dynamic system theory, organizing the sensorimotor system for movement involves interaction between sources of constraints, such as tasks, environments, and organisms. In this organization, network components are affected by inputs above the center of the brain (environmental inputs) when external disturbance and self-organizational constraints are imposed [11]. As an added restriction, people with CAI may be unable to create the self-organizational patterns necessary to deal with external disturbances [11]. This incidence of CAI means inadequate postural control, and maintaining such regulation requires accurate information from various sensory systems [12]. Sensory exchange theory maintains that when a sensory path- way is damaged or lost, in adapting to this impairment, the CNS heavily weighs remaining inputs and relies on these signals to generate motor responses. Reliance on one type of input may increase the risk of falls when excessive, continuous, or unreliable weight is attached to input information [13]. The results obtained by Riva et al. showed that progress in proprioceptive control in a single-leg position could be a significant factor for the effective reduction of ankle sprains, knee sprains, and chronic low back pain. This reduction operates through the mechanism of perceived progress in sustainability, technical skill, and motion control [14].

An issue related to appropriate posture control is static balance, defined as balance at a stable level without intentional movement by a person [15]. A static single-leg balance test is a reliable gauge of athletic performance. Because postural control measures are the most vital indicators of CAl, therefore, the Center Of Pressure (CoP) levels are frequently incorporated into analyses of postural control [16]. About CoP, one of the essential factors for executing sport skills is the level of balance in the preferred foot of athletes, especially elite players in sports fields such as volleyball and basketball $[3,6,7]$. Competencies such as shooting in soccer, layups in basketball, and take-offs to perform a volleyball spike are examples of the instrumentality of foot reliance among athletes $[3,6,7]$. Given this situation, there is a high prevalence of ankle sprains in the preferred foot of elite players of different sports [8]. Although these injuries are considerably alleviated through rehabilitation exercises, various reasons, including re-injury, the failure to complete a rehabilitation program, and the early initiation of exercise, leads to CAI in athletes, who are often compelled to continue participating in competitions while grappling with such impairments [9-11]. The majority of these elite players, thus, suffer from diminished accuracy and balance in the preferred foot, thereby disabling them to have high-quality performances same as before the injury. In this situation, despite the vital role of the visual sense in postural control, decreasing dependency in the visual sense may divert attention to other sensory inputs, especially proprioception, which is vital for sports balance and performance.

Considering the above-mentioned issues, we need to improve our understanding of balance in the preferred foot of elite players and CAI, as well as the role of proprioception and vision in these matters. This understanding can facilitate the design of more effective exercises that strengthen balance and increase accuracy in performing sports skills. With this background in mind, we compared elite soccer, volleyball, and basket- 
ball players with CAI in terms of balance performance in conditions with and without visual information. We hypothesized that the ability to control posture varied in professional athletes with chronic ankle instability. The obtained results can help set up a rehabilitation program after injury specifically and according to the needs of athletes in each sport.

\section{Material and Methods}

\section{Study participants}

In this cross-sectional study, a total of 45 elite players with CAI were assigned to soccer, volleyball, and basketball groups of 15 individuals each. The sample size was estimated using GxPower, with an effect size of 0.55 , a test power of 0.90 , and an alpha value of 0.05 [17]. This research was approved by the Ethics Committee in Research. The subjects were selected from among the elite players of Premier League teams of soccer, volleyball, and basketball. The inclusion criteria, which were based on the standards approved by the International Ankle Consortium, were as follows: more than two incidences of ankle sprains or a greater need for medical treatment of the condition, feelings of fear and instability in ankle function, the collapse of the foot during physical activities, a score of $\leq 24$ in the Cumberland Ankle Instability Tool (CAIT), and the confirmation of CAI via anterior drawer and talar tilt tests performed by an experienced physician [18]. The athletes recruited for participation should have had CAI on one foot only, with the affected leg as the dominant one [8]. The exclusion criteria were a history of surgery on the lower extremity and a history of fractures and acute damage to the musculoskeletal structure or joints of the lower extremity (e.g. sprains and fractures) in the last three months, leading to missed physical activity for at least one day [18].

\section{Lab procedure}

Bu-Ali Sina University Research Ethics Committee approved this study (No.: IR.BASU.REC.1399.027). Written informed consent was obtained from the participants; then they were administered the CAIT and a questionnaire on the history of injury. Notably, the elite players reported instability in the preferred foot, which the elite soccer players use to score a goal. The weight and height of the participants were measured using a Biospace stadiometer (Korea), and they were instructed to perform a 10-min warmup before the start of a static single-leg balance test. To perform the test, the elite players were required to stand on their injured foot on a platform in a single-leg position for 20 seconds while keeping oscillations to a minimum. They were asked to put their hands on their hip and bend the other knee and pull it back. The tests were performed barefoot, and the participants were directed to look at the target located before them $2 \mathrm{~m}$ from the wall. The test was also carried out with the participants' eyes closed. In total, three successful trials (for both open- and closedeye situations) were recorded for each participant, with a 30-s rest allowed between tests. Test errors included the separation of hands from the hip, contact between the non-preferred foot and the ground, and the movement of the preferred foot during the tests $[3,6]$.

\section{Outcome variables}

A plantar pressure platform (FDM-S, Zebris Medical $\mathrm{GmbH}$, Germany) composed of 2560 high-sensitivity sensors and an acquisition frequency of $120 \mathrm{~Hz}$ was used to record the test performance (Figure 1). The Zebris platform has good validity and reliability, which have been reported in previous studies [19-21]. The CoP oscillation parameters covered the following items:

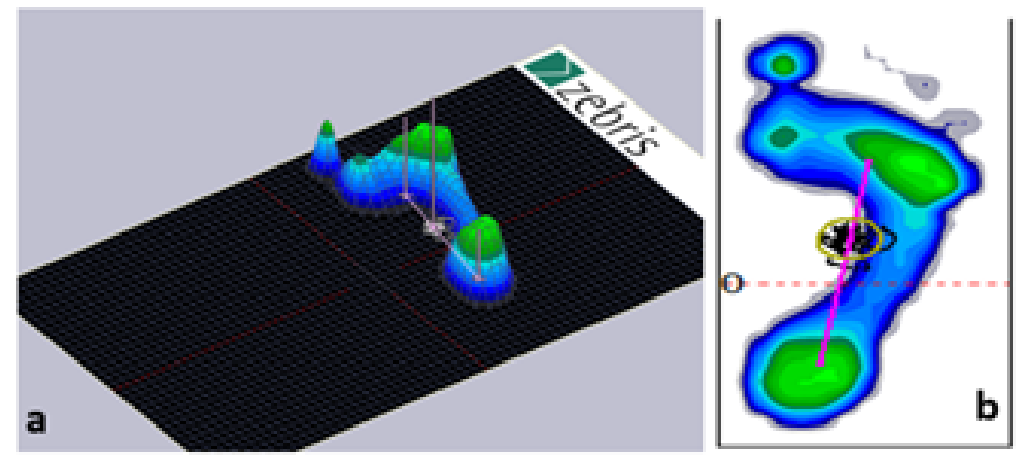

Figure 1. A plantar pressure platform

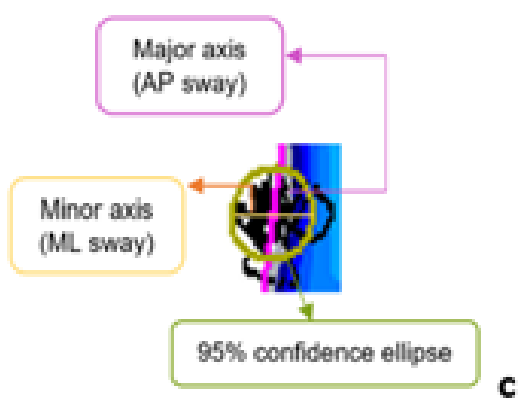

Exercise Science and Medicine

$A: 3 D$ foot pressure single leg stance; $B: 2 D$ foot pressure single leg stance; $C$ : COP oscillation parameters. 
- CoP path length (CoP PL) (the overall distance traveled by the CoP during a trial).

- CoP sway velocity (CoP SV)

- CoP displacement in the mediolateral (ML) (minor axis) and Anteroposterior (AP) (major axis) directions

- Sway Area (SA) (95\% confidence ellipse)

A: 3D foot pressure single-leg stance; $B: 2 D$ foot pressure single-leg stance; $\mathrm{C}$ : CoP oscillation parameters.

\section{Statistical analysis}

The data normality was examined using the ShapiroWilk test $(P>0.05)$. One-way analysis of variance (ANOVA) and Tukey test were conducted, using the SPSS (version 21). A P value less than 0.05 was considered indicative of statistical significance.

\section{Results}

Table 1 presents the athletes' characteristics. The results of the static open-eye, single-leg balance test indi- cated a significant difference among the groups in terms of displacement in AP sway (major axis) and ML sway (minor axis) as well as CoP SA. The Tukey test results also reflected a significant difference between the soccer and volleyball players in terms of the length of AP sway. The same statistically significant difference occurred between the soccer group and the volleyball and basketball groups concerning AP sway and CoP SA (Table 2).

The findings derived from the static closed-eye, singleleg balance test showed a significant difference among the groups concerning AP and ML sway and CoP SA. The Tukey test pointed to an equally significant difference between the soccer and volleyball groups in terms of AP and ML sway as well as CoP SA (Table 3).

Regardless of whether visual information was available, no significant difference was found among the groups regarding CoP PL and CoP SV (Table 3).

\section{Discussion}

As previously described, this research aimed at comparing the performance of elite players with CAI in a

Table 1. Athletes' characteristics

\begin{tabular}{cccc}
\hline Groups & \multicolumn{3}{c}{ Mean \pm SD } \\
\cline { 2 - 4 } & Soccer & Volleyball & Basketball \\
\hline Age (year) & $21.8 \pm 2.14$ & $22.33 \pm 2.35$ & $22.13 \pm 2.26$ \\
Height (cm) & $181.33 \pm 3.51$ & $182.73 \pm 4.46$ & $184.53 \pm 4.2$ \\
Body mass (kg) & $71.66 \pm 2.94$ & $72.06 \pm 3.71$ & $73.06 \pm 3.21$ \\
CAIT (score) & $20.46 \pm 1.4$ & $20.26 \pm 1.46$ & $20 \pm 1.46$ \\
\hline Ankle sprains (number of times) & $3.93 \pm 0.79$ & $3.80 \pm 0.86$ & $3.93 \pm 0.80$ \\
\hline
\end{tabular}

Table 2. One-way ANOVA results in relation to the static single-leg balance test performed with eyes open

Exercise Science and Medicine

\begin{tabular}{ccccccc}
\hline Items & Soccer & Volleyball & Basketball & F & P & Post Hoc \\
\hline ML sway $(\mathrm{mm})$ & $8.7 \pm 0.88$ & $11.58 \pm 3.01$ & $10.7 \pm 1.48$ & 8.13 & $0.001^{*}$ & $\begin{array}{c}\mathrm{S}<\mathrm{V} \\
\mathrm{S}<\mathrm{B}\end{array}$ \\
AP sway $(\mathrm{mm})$ & $14.91 \pm 2.42$ & $18.92 \pm 6.49$ & $16.1 \pm 2.9$ & 3.5 & $0.039^{*}$ & $\mathrm{~S}<\mathrm{B}$ \\
\hline $\mathrm{SA}(\mathrm{mm} \times \mathrm{mm})$ & $103.5 \pm 17.54$ & $177.24 \pm 82.54$ & $157.61 \pm 59.15$ & 6.57 & $0.003^{*}$ & $\mathrm{~S}<\mathrm{V}$ \\
$\mathrm{S}<\mathrm{B}$
\end{tabular}

ML: Mediolateral, AP: Anteroposterior, SA: Sway area, PL: Path length, SV: Sway velocity, S: Soccer, V: Volleyball, B: Basketball.

*Significant difference $(\mathrm{P}<0.05)$. 
Table 3. One-way ANOVA results in connection to the static single-leg balance test performed with eyes closed

\begin{tabular}{ccccccc}
\hline Items & Soccer & Volleyball & Basketball & F & P & Post Hoc \\
\hline ML sway $(\mathrm{mm})$ & $24.13 \pm 2.64$ & $28.5 \pm 5.4$ & $25.13 \pm 6.27$ & 2.98 & $0.05^{*}$ & $\mathrm{~S}<\mathrm{V}$ \\
AP sway $(\mathrm{mm})$ & $31.65 \pm 3.13$ & $42.98 \pm 11.45$ & $37.14 \pm 9.37$ & 6.64 & $0.003^{*}$ & $\mathrm{~S}<\mathrm{V}$ \\
$\mathrm{SA}(\mathrm{mm} \times \mathrm{mm})$ & $587.41 \pm 112.65$ & $1018.68 \pm 432.16$ & $792.38 \pm 425.37$ & 5.8 & $0.006^{*}$ & $\mathrm{~S}<\mathrm{V}$ \\
COP PL $(\mathrm{mm})$ & $1092.36 \pm 202.87$ & $1234.54 \pm 353.05$ & $1114.9 \pm 277.74$ & 1.11 & 0.33 \\
SV $(\mathrm{mm} / \mathrm{sec})$ & $58.83 \pm 11.18$ & $61.21 \pm 39.69$ & $63.44 \pm 20.44$ & 2.68 & 0.07 \\
\hline
\end{tabular}

ML: Mediolateral, AP: Anteroposterior, SA: Sway area, PL: Path length, SV: Sway velocity, S: Soccer, V: Volleyball.

* Significant difference $(P<0.05)$.

static single-leg balance test under conditions typified by the presence and absence of visual information. The results of the static open-eye, single-leg balance test indicated a significant difference among the soccer, volleyball, and basketball elite players in terms of ML oscillations and SA. A significant difference was also found between the soccer and volleyball elite players regarding AP sway. Nonetheless, no difference occurred among the groups in terms of CoP PL and the speed of oscillations. The injury occurs when the stress imposed on tissue is chronically or acutely greater than the ability of the tissue to "absorb" such pressure. In sports such as basketball, soccer, and volleyball, the first initial sprain injury is succeeded by a recurrent sprain in many people. Among these cases, $20 \%$ to $50 \%$ end in ankle instability [14]. Susceptibility to chronic disability and the early onset of ankle joint degenerative diseases are more important than an injury itself [16]. Besides joint instability that develops over time and exceeds the joint's physiological boundaries, the risk of damage to joint surfaces and osteoporosis development increases [11].

Proprioception and motion perception plays a vital role in the prevention of sports-related injuries. Defects in the perception of proprioception for various reasons are claimed to increase injuries during sports [5]. Maintaining balance while executing various sports maneuvers is essential for ensuring optimal performance, enhancing the quality of activity, and preventing injury. For instance, soccer players must maintain a balanced position when running at high speeds, change directions quickly, and powerfully pass the ball or score a goal. Players must also maintain balance as they are blocked by opposing players (defense) [2]. Basketball skills involve tremendous joint acceleration in landing jumps and cutting maneuvers [22]. Basketball players need to sustain postural control when landing on the ground following a fight to secure a rebound against an opposing player [5]. Meanwhile, although volleyball teams are separated by a net and play without contact, tasks specific to the sport, such as jumping, landing, defending, and spiking must be combined with fast movements. Volleyball players must also maintain their stability to prevent musculoskeletal injuries [23].

The effectiveness of balance training as a preventive measure against serious injuries has been demonstrated in various sports. Vertical position control (standing stability), for example, depends on the integration of information from the visual, vestibular, and proprioceptive systems [6]. People with CAI have impaired sensory-motor receptors (somatosensory), which reduces their motor response in maintaining postural control [24]. Moreover, it has been hypothesized that specific information from the lateral part of the ankle joint is primarily extracted by the receptors in lateral ligaments. Trauma to the ligaments by affecting mechanical receptors can trigger a misinterpretation of inverting ankle angle repetitions [25]. Athletes with CAl experience recurrent inverted ankle injuries in situations that do not arise during the physical performance with a stable ankle [25]. According to Wang et al., poor postural control is the best predictor of ankle sprains [26] Conversely, a short CoP displacement path and a low CoP speed reflect good postural control [16]. In general, a large CoP trajectory is interpreted as equivalent to poor stability [6].

In this work, the soccer players exhibited stronger static balance control than that showed by the volleyball and basketball players. Superior balance among elite players might result from repeated experience, which affects the motor responses and proprioceptive abilities of players and corresponding visual cues [7]. The better performance of the soccer players in this research may 
be attributed to the higher number of unilateral movements and the higher focus on the lower extremity in soccer than the activities involved in the two other sports. Soccer players perform various actions, such as scoring a goal, passing, and changing directions, most of which are unilateral. In contrast, volleyball and basketball players execute fewer unilateral movements in a game. Typically, soccer players pass, shoot, and dribble with their lower extremities with or without cleats in changing grass conditions $[3,22]$.

The environmental skills and demands associated with these sports are likely to pose several motor systemrelated challenges, whose repetition may affect the balancing competencies of players [22]. Although the lower extremity is used to dribble, pass, and shoot in basketball and spike, dig, and initiate a set in volleyball, these actions are performed with greater emphasis on the upper extremity. In terms of improved unilateral balance in the soccer players, our findings are in line with the results obtained by other researchers [7, 22, 27, 28]. According to sensory exchange theory, a person relies more on proprioceptive information to maintain balance by eliminating visual feedback when performing a test with eyes closed [4]. In the present study, however, slightly different results were obtained in the closedeye test. Considerable oscillations were observed in the three groups, but the performance of the basketball and soccer players in the open-eye test had no significant difference from their performance in the closed-eye test. This finding might be due to the appropriate compensation for inputs by other afferent nerves (proprioception) in the absence of visual information. Elite players are mainly trained to keep their eyes open, so performing a test with their eyes closed is challenging. Although complicated, the visual system can be typically divided into two essential components: the foveal component, which is mainly related to identifying objects, textures, and colors, and the peripheral vision, which is involved in detecting movements that occur in the surrounding environment. These neurologically and physiologically distinct components are implicated in postural control. In a standing position, for example, any change in the surrounding environment (position of objects or brightness level) affects postural oscillation [1].

The findings also reflected that the volleyball players more frequently depended on visual information than the other athletes. Elite volleyball players constantly use their visual system, as is the case with many other sports, but volleyball athletes perform eye movements in a particularly regular manner. Focus on players' eye positions when they are on the defensive underscores the impor- tance of proper eye movement in executing appropriate protective moves against an attack from an opposing team. The continuous ball movement trajectory on the playing field requires training of the eye muscles. As observed in the vision elimination test results, this "ocular training" affects postural control [6]. As demonstrated in Sarto et al. study, volleyball players experience recurrent injuries in the lower extremity, which adversely affect their balance. Therefore, maintaining stability is vital in several volleyball actions (e.g. receiving a service, defense, digging, and setting), in which at least one player's foot is in contact with the ground [29]. In the balance test performed with eyes open, the soccer players exhibited better static single-leg balance than other players. However, volleyball players performed poorly in the balance test, requiring them to keep their eyes closed. Volleyball players rely more frequently on visual information for postural control when they are afflicted with CAI. Besides, the necessity of strengthening proprioception is higher among these individuals. Given the recurrent jumps and lands and the need to maintain a good balance after landing, volleyball coaches should design practices that reduce dependence on visual cues and strengthen proprioception by imposing more challenges on proprioceptive senses. This does not mean, however, that soccer and basketball players with CAI do not need balance practice. Overall, the results demonstrated the need for greater emphasis on the balance issue in volleyball players.

One of the drawbacks of this study was the exclusive focus on the injured foot, including both feet in the tests and analyses, which may have produced different results. Muscle strength may have acted as a balance factor, but this aspect of the body was not measured in this work. Another limitation was that only male elite players with CAI were assessed; again, the findings may have been different if female athletes had been covered in the sample.

\section{Conclusion}

This research uncovered that in the static open-eye, single-leg balance test, the soccer players with CAI had better execution than that presented by the volleyball and basketball players. However, the volleyball players showed inferior performance in the static closed-eye, single-leg balance test. Given the nature of volleyball and the necessity of a good balance in elite players, especially those with CAI, balance practices that challenge proprioception should be designed by coaches to minimize reliance on visual information. Special balance 
practices should also be included in the regular practice sessions of elite players with CAI.

\section{Ethical Considerations}

\section{Compliance with ethical guidelines}

The study was approved by the Research Ethics Committee of Bu-Ali Sina University, Hamedan (Code No.: IR.BASU.REC.1399.027)

\section{Funding}

This research did not receive any grant from funding agencies in the public, commercial, or non-profit sectors.

\section{Authors' contributions}

Conceptualization, writing - review \& editing: Both authors; Methodology, software, data collection, writing - original draft preparation: Zahra Raeisi; Project administration: Ali Yalfani.

\section{Conflict of interest}

The authors declared no conflict of interest.

\section{References}

[1] Asseman FB, Caron O, Crémieux J. Are there specific conditions for which expertise in gymnastics could have an effect on postural control and performance? Gait Posture. 2008; 27(1):76-81. [DOI:10.1016/j.gaitpost.2007.01.004] [PMID]

[2] Erkmen N, Taşkın H, Sanioğlu A, Kaplan T, Baştürk D. Relationships between balance and functional performance in football players. Journal of Human Kinetics. 2010; 26(1):21-29. [DOI:10.2478/ v10078-010-0044-z]

[3] Jadczak Ł, Grygorowicz M, Wieczorek A, Śliwowski R. Analysis of static balance performance and dynamic postural priority according to playing position in elite soccer players. Gait Posture. 2019; 74:148-53. [DOI:10.1016/j.gaitpost.2019.09.008] [PMID]

[4] Ueta Y, Matsugi A, Oku K, Okuno K, Tamaru Y, Nomura S, et al. Gaze stabilization exercises derive sensory reweighting of vestibular for postural control. The Journal of Physical Therapy Science. 2017; 29(9):1494-6. [DOI:10.1589/jpts.29.1494] [PMID] [PMCID]

[5] Bayram M, Bayraktar G, Akyol H, Tozoğlu E. Comparison of Balance Performance of Elite Soccer Players and Skiers. International Journal of Sports Science. 2017; 7(1):10-14. http://article.sapub.org/10. 5923.j.sports.20170701.03.html

[6] Agostini V, Chiaramello E, Canavese L, Bredariol C, Knaflitz M. Postural sway in volleyball players. Human Movement Science. 2013; 32(3):445-56. [DOI:10.1016/j.humov.2013.01.002] [PMID]
[7] Hrysomallis C. Balance ability and athletic performance. Sports Medicine. 2011; 41:221-32. [DOI:10.2165/11538560-00000000000000] [PMID]

[8] Barlow G, Donovan L, Hart JM, Hertel J. Effect of lace-up ankle braces on electromyography measures during walking in adults with chronic ankle instability. Physical Therapy in Sport. 2015; 16(1):1621. [DOI:10.1016/j.ptsp.2014.02.002] [PMID]

[9] Steib S, Zech A, Hentschke C, Pfeifer K. Fatigue-induced alterations of static and dynamic postural control in athletes with a history of ankle sprain. Journal of Athletic Training. 2013; 48(2):203-8. [DOI:10.4085/1062-6050-48.1.08] [PMID] [PMCID]

[10] Lin JZ, Lin YA, Lee HJ. Are Landing Biomechanics Altered in Elite Athletes with Chronic Ankle Instability. Journal of Sports Science and Medicine. 2019; 18(4):653-62. [PMID] [PMCID]

[11] Brown CN, Mynark R. Balance deficits in recreational athletes with chronic ankle instability. Journal of Athletic Training. 2007; 42(3):367-73. [PMCID] [PMID]

[12] Yalfani A, Asgarpour A, Raeisi Z. Comparing the effective interventions on plantar and ankle proprioception in balance control with and without fatigue. Journal of Shahrekord University of Medical Sciences. 2018; 21(1):45-50. [DOI:10.34172/jsums.2019.08]

[13] Kabbaligere R, Lee BC, Layne CS. Balancing sensory inputs: Sensory reweighting of ankle proprioception and vision during a bipedal posture task. Gait Posture. 2017; 52:244-50. [DOI:10.1016/j. gaitpost.2016.12.009] [PMID]

[14] Riva D, Bianchi R, Rocca F, Mamo C. Proprioceptive training and injury prevention in a professional men's basketball team: A six-year prospective study. The Journal of Strength and Conditioning Research. 2016; 30(2):461-75. [DOI:10.1519/JSC.0000000000001097] [PMID] [PMCID]

[15] Arnold BL, De La Motte S, Linens S, Ross SE. Ankle instability is associated with balance impairments: A meta-analysis. Medicine \& Science in Sports \& Exercise. 2009; 41(5):1048-62. [DOI:10.1249/ MSS.0b013e318192d044] [PMID]

[16] Sefton JEM, Hicks-Little CA, Hubbard TJ, Clemens MG, Yengo $\mathrm{CM}$, Koceja DM, et al. Sensorimotor function as a predictor of chronic ankle instability. Clinical Biomechanics. 2009; 24(5):451-8. [DOI:10.1016/j.clinbiomech.2009.03.003] [PMID]

[17] Bhalerao S, Kadam P. Sample size calculation. International Journal of Ayurveda Research. 2010; 1(1):55. [DOI:10.4103/09747788.59946] [PMID] [PMCID]

[18] Gribble PA, Delahunt E, Bleakley CM, Caulfield B, Docherty CL, Fong DTP, et al. Selection criteria for patients with chronic ankle instability in controlled research: A position statement of the international ankle consortium. Journal of Athletic Training. 2014 49(1):121-7. [DOI:10.4085/1062-6050-49.1.14] [PMID] [PMCID]

[19] Chun HL, Lee BH. The immediate effects of foot pressure based insole on ankle dorsiflexion range of motion, postural sway, and muscle activation in healthy adults with genu varum. Physical Therapy Rehabilitation Science. 2018; 7(4):164-71. [DOI:10.14474/ ptrs.2018.7.4.164]

[20] Bae Y. Comparison of postural sway, plantar cutaneous sensation according to saccadic eye movement frequency in young adults. International Journal of Environmental Research and Public Health. 2020; 17(19):1-8. [DOI:10.3390/ijerph17197067] [PMID] [PMCID] 
[21] Nagymáté G, Orlovits Z, Kiss RM. Reliability analysis of a sensitive and independent stabilometry parameter set. PLoS One. 2019; 13(4):e0195995. [DOI:10.1371/journal.pone.0195995] [PMID] [PMCID]

[22] Bressel E, Yonker JC, Kras J, Heath EM. Comparison of static and dynamic balance in female collegiate soccer, basketball, and gymnastics athletes. Journal of Athletic Training. 2007; 42(1):42-6. [PMCID] [PMID]

[23] Kilic O, Maas M, Verhagen E, Zwerver J, Gouttebarge V. Incidence, aetiology and prevention of musculoskeletal injuries in volleyball: A systematic review of the literature. European Journal of Sport Science. 2017; 17(6):765-93. [DOI:10.1080/17461391.2017.1306114 ] [PMID]

[24] De Noronha M, Refshauge KM, Crosbie J, Kilbreath SL. Relationship between functional ankle instability and postural control. Journal of Orthopaedic \& Sports Physical Therapy. 2008; 38(12):782-9. [DOI:10.2519/jospt.2008.2766] [PMID]

[25] Konradsen L. Factors contributing to chronic ankle instability: Kinesthesia and joint position sense. Journal of Athletic Training. 2002; 37(4):381-5. [PMCID] [PMID]

[26] Wang HK, Chen $\mathrm{CH}$, Shiang TY, Jan MH, Lin KH. Risk-factor analysis of high school basketball-player ankle injuries: A prospective controlled cohort study evaluating postural sway, ankle strength, and flexibility. Archives of Physical Medicine and Rehabilitation. 2006; 87(6):821-5. [DOI:10.1016/j.apmr.2006.02.024] [PMID]

[27] Matsuda S, Demura S, Uchiyama M. Centre of pressure sway characteristics during static one-legged stance of athletes from different sports. Journal of Sports Sciences. 2008; 26(7):775-9. [DOI:10.1080/02640410701824099] [PMID]

[28] Thorpe JL, Ebersole KT. Unilateral balance performance in female collegiate soccer athletes. The Journal of Strength and Conditioning Research. 2008; 22(5):1429-33. [DOI:10.1519/ JSC.0b013e31818202db] [PMID]

[29] Sarto F, Grigoletto D, Baggio E, Paoli A, Marcolin G. Do lower limb previous injuries affect balance performance? An observational study in volleyball players. Physical Therapy in Sport. 2019; 37:49-53. [DOI:10.1016/j.ptsp.2019.02.009] [PMID] 